\title{
Computational simulation of the seismic response of buildings with energy dissipating devices
}

\author{
Alex H. Barbat, Pablo Mata \& Sergio Oller \\ Technical University of Catalonia, Barcelona, Spain \\ Juan C. Vielma \\ Lisandro Alvarado University, Barquisimeto, Venezuela
}

\begin{abstract}
In this work, the nonlinear dynamic response of RC buildings with energy dissipating devices is studied using advanced computational techniques. A fully geometric and constitutive nonlinear model is used for describing the dynamic behavior of structures. The equations of motion are expressed in terms of cross sectional forces and strains and its weak form is solved using the displacement based finite element method. A suitable version of Newmark's scheme is used in updating the kinematics variables in a classical Newton type iterative scheme. Material points of the cross section are assumed to be composed of several simple materials with their own constitutive laws. The mixing theory is used to treat the resulting composite. A specific finite element based on the beam theory is proposed for the dissipators including constitutive relations. Finally, several numerical tests are carried out to validate the proposed model.
\end{abstract}

\section{Introduction}

Conventional seismic design practice permits designing reinforced concrete (RC) structures for forces lower than those expected from the elastic response on the premise that the structural design assures significant structural ductility Hanson et al. (1993). Frequently, the dissipative zones are located near the beam-column joints and, due to cyclic inelastic incursions during earthquakes, several structural members can suffer a great amount of damage. This situation is generally considered economically acceptable if life safety and collapse prevention are achieved.

In the last decades, new techniques based on adding devices to the buildings with the main objective of dissipating the energy exerted by the earthquake and alleviating the ductility demand on primary structural elements have improved the seismic behavior of the structures Soong \& Dargush (1997). The purpose is to control the seismic response of the buildings by means of a set of dissipating devices which constitutes the control system, adequately located in the structure. In the case of passive energy dissipating devices (EDD), an important part of the energy input is absorbed and dissipated; therefore, concentrating the nonlinear phenomenon in the devices without the need of an external energy supply.

Several works showing the ability of EDDs in controlling the seismic response of structures are available; for example, in reference Fu \& Kasai (2002) the response of 
framed structures equipped with viscoelastic and viscous devices is compared; in reference Kasai et al. (1998) an approximated method is used to carry out a comparative study considering metallic and viscous devices. Aiken (1996) presents the contribution of the extra energy dissipation due to EDDs as an equivalent damping added to the linear bare structure and gives displacement reduction factors as a function of the added damping ratio. A critical review of reduction factors and design force levels can be consulted in reference Lin \& Chang (2003). A method for the preliminary design of passively controlled buildings is presented in reference Connor et al. (1997). Lin \& Chopra (2003) study the accuracy of estimating the dynamic response of asymmetric buildings equipped with EDDs, when they are replaced by their energetic equivalent viscous dampers. Other procedures for the analysis and design of structures with EDDs can be consulted in reference Clark et al. (1999).

Today, only a few countries have codes to design RC buildings with EDDs. Particularly, in United States the US Federal Emergency Management Agency (FEMA) gives code provisions and standards for the design of EDDs devices to be used in buildings. In Europe, the efforts have been focused on developing codes for base isolation but not for the use of EDDs.

The design methods proposed for RC structures are mainly based on supposing that the behavior of the bare structure remains elastic, while the energy dissipation relies on the control system. However, experimental and theoretical evidence show that

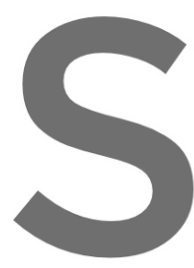
inelastic beharig

(Shen \& Soeng 2005 )

passively contro

both academics

There is agreement that

most precise tools for th
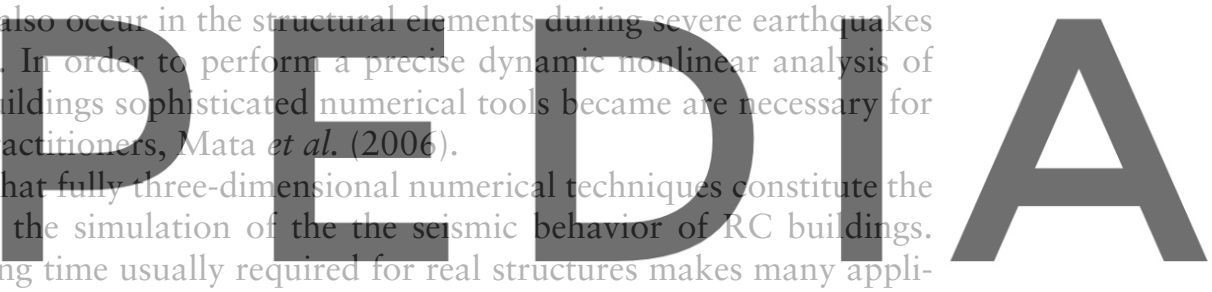

cations unpractical. Considering that most of the elements in RC buildings are columns

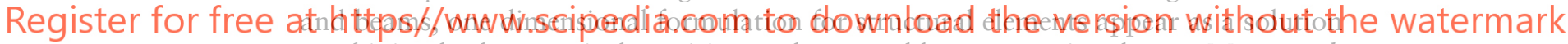
combining both numerical precision and reasonable computational costs Mata et al. (2007b). Experimental evidence shows that inelasticity in beam elements can be formulated in terms of cross sectional quantities, Bayrak et al. (2001). Some formulations of this type have been extended for considering geometric nonlinearities, Simo et al. (1984). An additional refinement is obtained considering inhomogeneous distributions of materials on arbitrarily shaped beam cross sections, Kumar et al. (2004). In this case, the constitutive relationship at cross sectional level is deduced by integration and, therefore, the mechanical behavior of beams with complex combinations of materials can be simulated.

Formulations for beams considering both constitutive and geometric nonlinearity are rather scarce; most of the geometrically nonlinear models are limited to the elastic case, Ibrahimbegovic (1995) and the inelastic behavior has been mainly restricted to plasticity, Simo et al. (1984). Recently, Mata et al. (2007b, 2008a) have extended the geometrically exact formulation for beams due to Reissner-Simo (Reissner 1973, Simo 1985, Simo \& Vu-Quoc 1988) to an arbitrary distribution of composite materials on the cross sections for the static and dynamic cases.

From the numerical point of view, EDDs usually have been described in a global sense by means of force-displacement or moment-curvature relationships, Soong \& Dargush 
(1997), which attempt to capture appropriately the energy dissipating capacity of the devices (see Mata et al. 2007a and 2008b). The inclusion of EDDs in software packages for the seismic analysis of RC structures is frequently done by means of linking elements equipped with the mentioned nonlinear relationships. The relative displacement and/or rotation between the anchorage points activate the dissipative mechanisms of the device.

In this work, a fully geometric and constitutive nonlinear formulation for beam elements is developed. A fiber-like approach is used for representing arbitrary distributions of composite materials on the plane beam cross sections. EDDs are considered as beam elements without rotational degrees of freedom. Thermodynamically consistent constitutive laws are used for concrete, longitudinal and transversal steel reinforcements and EDDs. The mixing rule is employed for the treatment of the resulting composite. A brief description of the damage indices capable of estimate the remaining load carrying capacity of the buildings is also given. Finally, the results obtained from numerical simulations showing the ability of the proposed formulation in simulating the static and dynamic inelastic response of RC buildings with and without EDDs are provided.

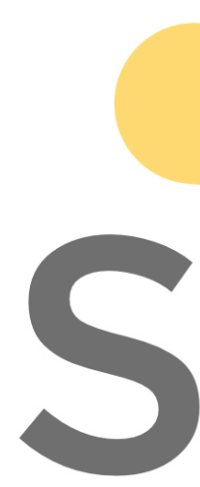

2 Finite deformation formulation for structural elements

\section{B Beam model}

The original geo (1986) is exp ration accorditg
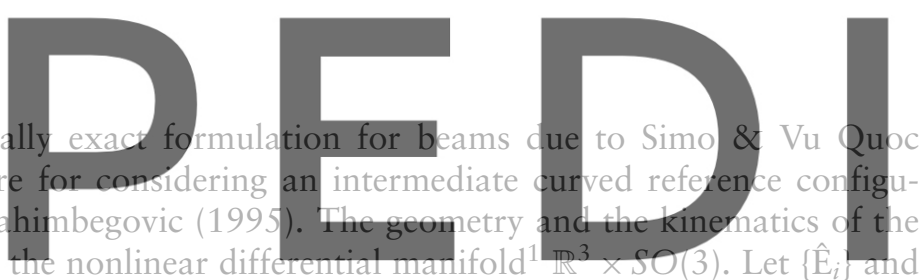

$\left\{\hat{e}_{i}\right\}$ be the spatially fixed material and spatial frames², respectively. The straight refer-

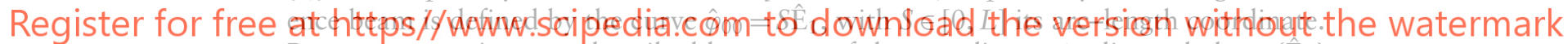
Beam cross sections are described by means of the coordinates $\xi_{\beta}$ directed along $\left\{\hat{E}_{\beta}\right\}$ and the position vector of any material point is $\hat{X}=S \hat{E}_{1}+\Sigma_{\beta} \xi_{\beta} \hat{E}_{\beta}$.

The curved reference beam is defined by means of the spatially fixed curve given by $\hat{\varphi}_{0}=\Sigma_{i} \hat{\varphi}_{0 i}(S) \hat{\mathrm{e}}_{i} \in \mathbb{R}^{3}$. Additionally, each point on this curve has rigidly attached an orthogonal local frame $\hat{t}_{0 i}(S)=\boldsymbol{\Lambda}_{0} \hat{\mathrm{E}}_{i} \in \mathbb{R}^{3}$, where $\boldsymbol{\Lambda}_{0} \in S O(3)$ is the orientation tensor. The beam cross section $A(S)$ is defined considering the local coordinate system $\xi_{\beta}$ but directed along $\hat{t}_{0 \beta}$. The planes of the cross sections are normal to the vector tangent to the reference curve ${ }^{3}$, i.e. $\hat{\varphi}_{0, s}=\hat{t}_{01}(S)$. The position vector of a material point on the curved reference beam is $\hat{x}_{0}=\hat{\varphi}_{0 i}+\Sigma_{\beta} \boldsymbol{\Lambda}_{0} \xi_{\beta} \hat{\mathrm{E}}_{0 \beta}$. The motion deforms points on the curved reference beam from $\hat{\varphi}_{0, S}$ to $\hat{\varphi}_{S, t}$ (at time $t$ ) adding a translational displacement $\hat{u}(S)$ and the local orientation frame is simultaneously rotated together with the beam cross section, from $\boldsymbol{\Lambda}_{0}(S)$ to $\boldsymbol{\Lambda}(S, t)$ by means of the incremental rotation tensor as $\boldsymbol{\Lambda}=\boldsymbol{\Lambda}_{n} \boldsymbol{\Lambda}_{0} \equiv \Sigma_{i} \hat{t}_{i} \otimes \hat{\mathrm{E}}_{i} \in S O(3)$ (see Figure 1).

\footnotetext{
${ }^{1}$ The symbol $S O(3)$ is used to denote the finite rotation manifold

${ }^{2}$ The indices $i$ and $\beta$ range over and $\{1,2,3\}$ and $\{2,3\}$, respectively.

${ }^{3}$ The symbol $(\bullet)_{x}$ is used to denote partial differentiation of $(\bullet)$ with respect to $x$.
} 

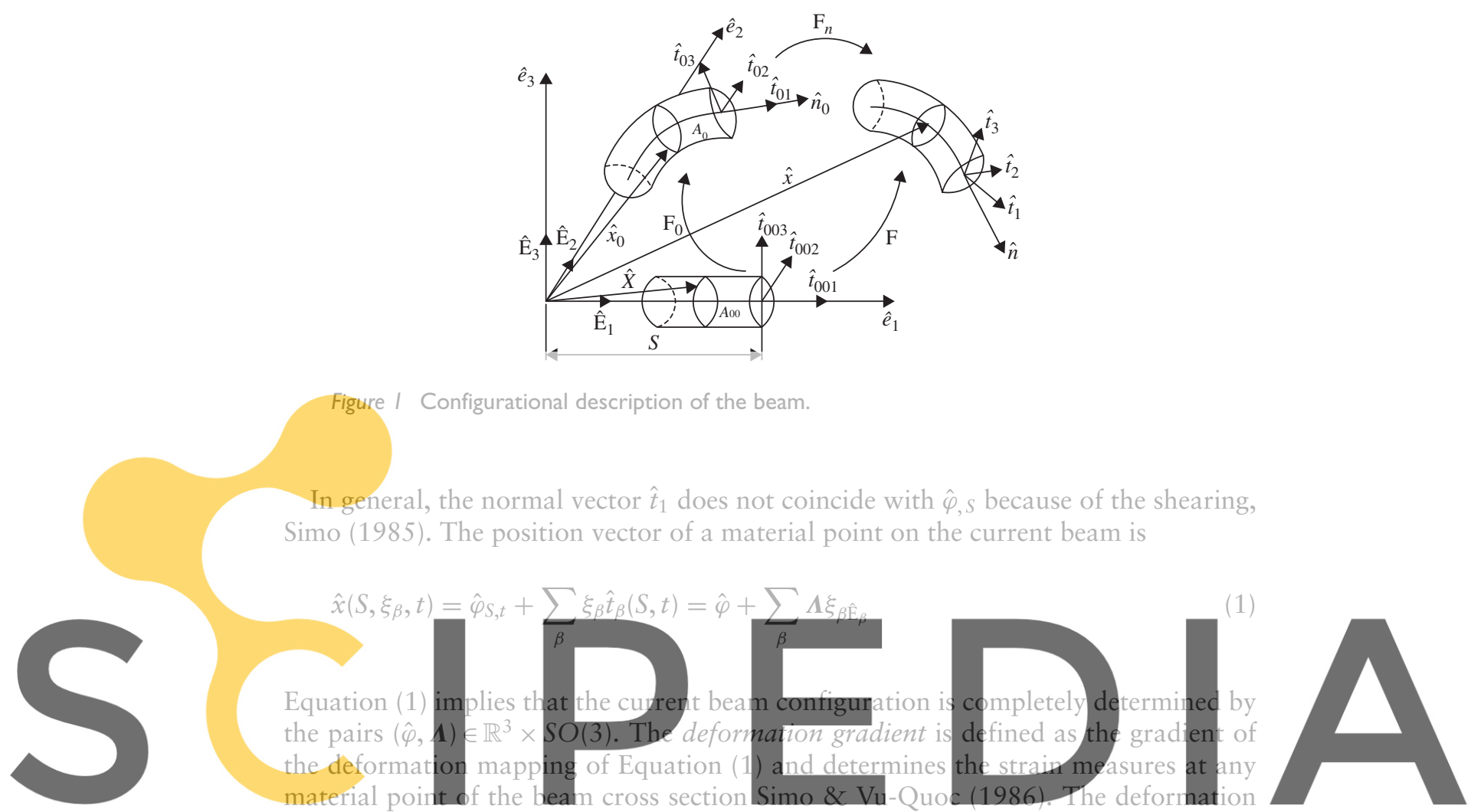

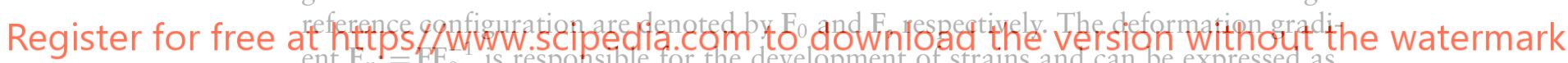
Kapania (2003) and Mata et al. (2007).

$$
\mathbf{F}_{n}=\mathbf{F F}_{0}^{-1}=\frac{1}{\left|\mathbf{F}_{0}\right|}\left[\hat{\varphi}_{, S}-\hat{t}_{1}+\tilde{\boldsymbol{\omega}}_{n} \sum_{\beta} \xi_{\beta} \hat{t}_{\beta}\right] \otimes \hat{t}_{01}+\boldsymbol{\Lambda}_{n}
$$

where $\left|\mathbf{F}_{0}\right|$ is the determinant of $\mathbf{F}_{0}$ and $\tilde{\boldsymbol{\omega}}_{n}=\boldsymbol{\Lambda}_{n, S} \boldsymbol{\Lambda}_{n}^{T}$ is the curvature tensor relative to the curved reference beam. In Equation (2) the term defined as $\tilde{\gamma}_{n}=\hat{\varphi}_{, S}-\hat{t}_{1}$ corresponds to the reduced strain measure of shearing and elongation, Kapania (2003) and Simo (1985), with material description given by $\hat{\Gamma}=\boldsymbol{\Lambda}^{T} \tilde{\gamma}$. The material representation of $\mathbf{F}_{n}$ is obtained as $\mathbf{F}_{n}^{m}=\boldsymbol{\Lambda}^{T} \mathbf{F}_{n} \boldsymbol{\Lambda}_{0}$.

Removing the rigid body component from $\mathrm{F}_{n}$, it is possible to construct the strain tensor $\varepsilon_{n}=\mathbf{F}_{n}-\boldsymbol{\Lambda}_{n}$, which conjugated to the asymmetric First Piola Kirchhoff (FPK) stress tensor $P=\hat{P}_{i} \otimes \hat{t}_{0 i}$ referred to the curved reference beam, Simo (1985). $\hat{P}_{i}$ is the FPK stress vector acting on the deformed face in the current beam corresponding to the normal $\hat{t}_{0 i}$ in the curved reference configuration. The spatial strain vector acting on the current beam cross section is obtained as $\hat{\varepsilon}_{n}=\varepsilon_{n} \hat{t}_{01}$. 
By other hand, the spatial form of the stress resultant $\hat{n}$ and the stress couple $\hat{m}$ vectors can be estimated from the stress vector $\hat{P}_{1}$ according to

$$
\hat{n}(S)=\int_{A} \hat{P}_{1} d A ; \quad \hat{m}(S)=\int_{A}(\hat{x}-\hat{\varphi}) \hat{P}_{1} d A ;
$$

The material form of $\hat{P}_{j}$ and $\varepsilon_{n}$ are obtained by means of the pull-back operation as $\hat{\varepsilon}_{n}=\boldsymbol{\Lambda}^{T} \varepsilon_{n}, \hat{P}_{j}^{m}=\boldsymbol{\Lambda}^{T} \hat{P}_{j}, \hat{n}^{n}=\boldsymbol{\Lambda}^{T} \hat{n}$ and $\hat{m}^{n}=\boldsymbol{\Lambda}^{T} \hat{m}$ respectively.

An objective measure of the strain rate vector $s_{n}$ acting on any material point on the current beam cross section can be deduced following the results presented by Mata et al. (2008a) and using the definition of the Lie derivative operator $[\bullet]$ given in Mata et al. (2007b) as follows:

$$
s_{n}=\left[\begin{array}{l}
\nabla \\
\hat{\varepsilon}_{n}
\end{array}\right]=\left[\hat{\hat{\gamma}}_{n}\right]+\left[\dot{\tilde{\omega}}_{n}\right] \sum_{\beta} \xi_{\beta} \hat{t}_{\beta}=\hat{\varphi}, S-\hat{v}_{n} \tilde{\varphi}_{, S}+\hat{v}_{n, S} \sum_{\beta} \xi_{\beta} \hat{t}_{\beta}
$$

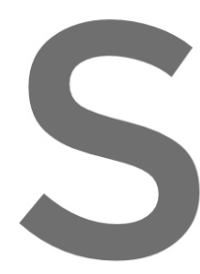

where $\tilde{v}_{n}=\dot{\Lambda}_{n} \Lambda_{n}^{T}$ is the current spin or angular velocity of the beam cross section with
respect to the curved reference beam. The material form of Equation (4) is $\hat{S}_{n}=\Lambda^{T} \hat{s}_{n}$. According to the developments given by Antman (1991), the classicol form equations of motion of the Cosserat beam
\[ \hat{n}_{S}+\hat{n}_{p}=A_{p 0} \ddot{\hat{\varphi}}+\underbrace{\tilde{\alpha}_{n} \hat{S}_{\rho 0}+\tilde{\boldsymbol{v}}_{n} \tilde{v}_{n} \hat{S}_{\rho 0}}_{D_{1}} \]
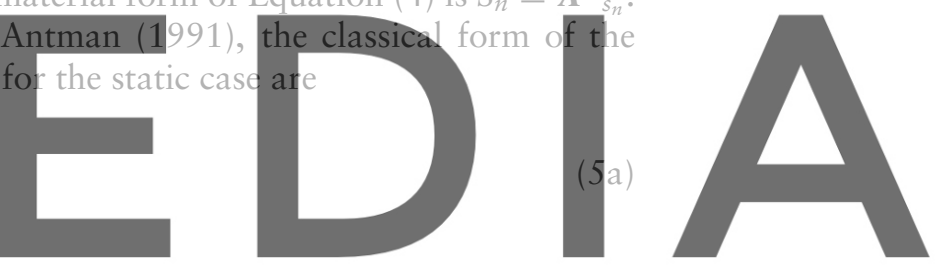

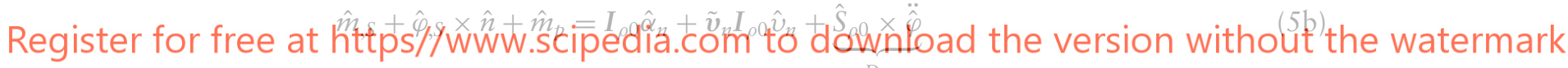

where $\hat{n}_{p}$ and $\hat{m}_{p}$ are the external body force and body moment per unit of reference length at time $t, A_{\rho 0}, \hat{S}_{\rho 0}$ and $I_{\rho 0}$ are the cross sectional mass density, the first mass moment density and the second mass moment density per unit of length of the curved reference beam, respectively; their explicit expressions can be consulted in Kapania (2003) and Simo \& Vu-Quoc (1986). $\tilde{\boldsymbol{\alpha}}_{n} \equiv \ddot{\boldsymbol{\Lambda}}_{n} \boldsymbol{\Lambda}_{n}^{T}-\tilde{\boldsymbol{v}}_{n}^{2}$ is the angular acceleration of the beam cross section and $\hat{v}_{n}$ and $\hat{\alpha}_{n}$ are the axial vectors of $\tilde{\boldsymbol{v}}_{n}$ and $\tilde{\boldsymbol{\alpha}}_{n}$, respectively. For most of the practical cases, the terms $D_{1}$ and $D_{2}$ can be neglected or added to the external forces and moments.

Considering a kinematically admissible variation ${ }^{4} \hat{b} \equiv(\delta \hat{\varphi}, \delta \hat{\theta})$ of the pair $(\hat{\varphi}, \boldsymbol{\Lambda})$, taking the dot product with Eqs. (5a) and (5b), integrating over the length of the curved reference beam and integrating by parts, we obtain the nonlinear functional $G(\hat{\varphi}, \boldsymbol{\Lambda}, h)$ corresponding to the weak form of the balance equations, Ibrahimbegovic (1995) and

\footnotetext{
${ }^{4}$ Supposing that $\boldsymbol{\Lambda}$ is parameterized in terms of the spatial rotation vector and following the results of reference it is possible to show that $\delta \boldsymbol{\Lambda}=\delta \hat{\theta} \times \boldsymbol{\Lambda}$ with $\delta \hat{\theta}$ an admissible variation of the rotation vector.
} 
Simo \& Vu-Quoc (1986), which is another way of writing the virtual work principle,

$$
\begin{aligned}
G(\hat{\varphi}, \boldsymbol{\Lambda}, h)= & \int_{L}\left[(\delta \hat{\varphi}, S-\delta \hat{\theta} \times \hat{\varphi}, S) \cdot \hat{n}+\delta \hat{\theta}_{, S} \cdot \hat{m}\right] d S \\
& +\int_{L}\left[\delta \hat{\varphi} A_{\rho 0} \ddot{\hat{\varphi}}+\delta \hat{\theta} \cdot\left(I_{\rho 0} \hat{\alpha}_{n}+\tilde{\boldsymbol{v}}_{n} I_{\rho 0} \hat{v}_{n}\right)\right] d S \\
& -\int_{L}\left[\delta \hat{\varphi} \cdot \hat{n}_{p}+\delta \hat{\theta} \cdot \hat{m}_{p}\right] d S-\left.(\delta \hat{\varphi} \cdot \hat{n}+\delta \hat{\theta} \cdot \hat{m})\right|_{0} ^{L}=0
\end{aligned}
$$

The terms $(\delta \hat{\varphi}, S-\delta \hat{\theta} \times \hat{\varphi}, S)$ and $\delta \hat{\theta}, S$ appearing in Equation (6) correspond to the co-rotated variations of the reduced strain measures $\hat{\gamma}_{n}$ and $\hat{\omega}_{n}$ in spatial description.

\subsection{Energy dissipating devices}

The finite deformation model for EDDs is obtained from the beam model releasing the rotational degrees of freedom and supposing that all the mechanical behavior of the device is described in terms of the evolution of a unique material point in the middle of the resulting bar.

The current position of a point in the EDD bar is obtained from Equation (1) and considering that $\xi_{\beta}=0$ as $\hat{x}(S, t)=\hat{\varphi}(S, t)$. Supposing that the current orientation of the

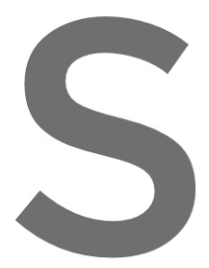
EDD bar of initial lengt
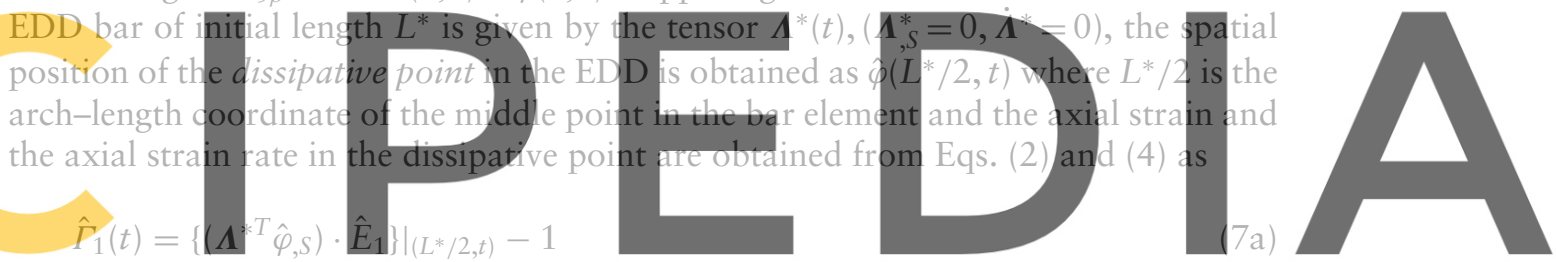

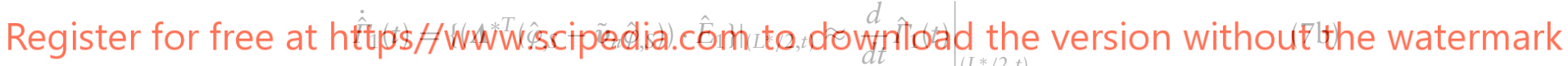

Finally, the contribution of the EDD bar to the functional of Equation (6), written in the material description, is given by

$$
G_{E D D}=\int_{L *} n_{1}^{m} \delta \hat{\Gamma}_{1} d S+\left.\left\{\left(\boldsymbol{\Lambda}^{* T} \delta \hat{\varphi}\right)^{T}[\boldsymbol{M}]_{d}\left(\boldsymbol{\Lambda}^{* T} \ddot{\hat{\varphi}}\right)\right\}\right|_{\left(L^{*} / 2, t\right)}
$$

where it was assumed that $I_{\rho 0} \approx 0$, i.e. the contribution of the EDDs to the rotational mass of the system is negligible and $[M]_{d}$ is the EDD's translational inertia matrix, i.e. the mass of the control system is supposed to be concentrated on the central point of the bar. The term $\delta \hat{\Gamma}_{1}=\left(\boldsymbol{\Lambda}^{* T}\left(\hat{\varphi}_{, S}-\tilde{\boldsymbol{v}}_{n} \hat{\varphi}_{, S}\right)\right) \cdot \hat{E}_{1}$ corresponds to the material form of the variation of the axial strain in the EDD.

\section{Constitutive models}

In this work, material points on the cross sections are considered as formed by a composite material corresponding to a homogeneous mixture of different simple components, each of them with its own constitutive law (see Figure 2). The resulting 


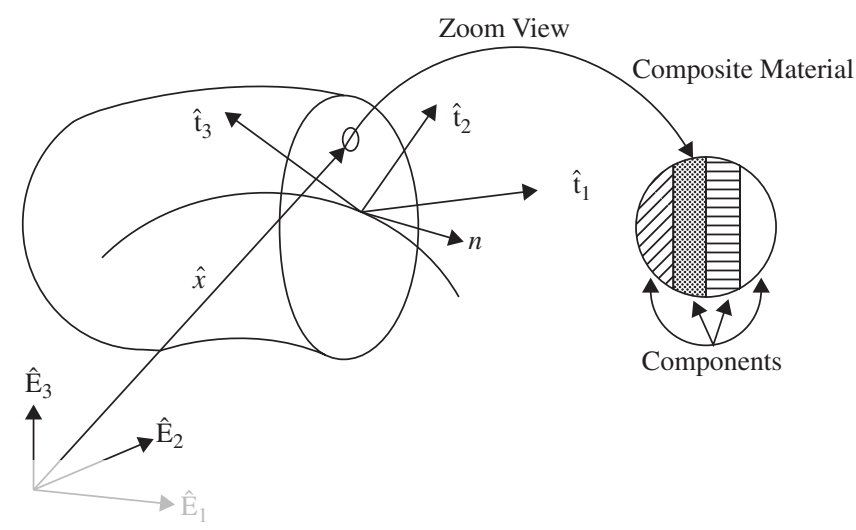

Figure 2 Cross section showing the composite associated to a material point.

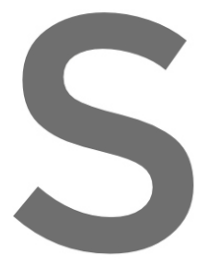

behavior is obtained by means of the mixing theory. Two kinds of nonlinear consti-
tutive models for simple materials are used: the damage and plasticity models. The constitutive models are formulated in terms of the material form of the FPK stress and strain vectors, $\hat{P}_{1}^{m}$ and $\hat{\varepsilon}_{n}$, respectively, Mata et al. (2007b and 2008a)

3.l Degrading materials: damage

The damage ther function which differentlates the mechanic

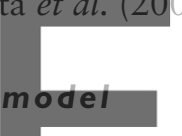

ponents of the stress vector. The progress of the damage is based on the evolution of

a scalar damage parameter Oliver et al. (1990). Starting from an adequate form of

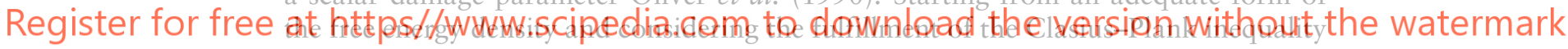

and applying the Coleman's principle (see Mata et al. 2007b and 2008c) the following

constitutive relation in material form is obtained:

$$
\hat{P}_{1}^{m}=(1-d) \mathcal{C}^{m e} \hat{\varepsilon}_{n}=\mathcal{C}^{m s} \hat{\varepsilon}_{n}=(1-d) \hat{P}_{01}^{m}
$$

where $\mathcal{C}^{m e}$ and $\mathcal{C}^{m s}=(1-d) \mathcal{C}^{m e}$ is the secant constitutive tensor. Equation (9) shows that the FPK stress vector is obtained from its elastic counterpart by multiplying it by the factor $(1-d)$.

The damage yield criterion $\mathcal{F}$, Hanganu et al. (2003) and Barbat et al. (1997), is defined as a function of the undamaged elastic free energy density and written in terms of the components of the material form of the undamaged principal stresses, $\hat{P}_{01}^{m}$, as

$$
\mathcal{F}=\mathcal{P}-f_{c}=[1+r(n-1)] \sqrt{\sum_{i=1}^{3}\left(P_{\rho 0 i}^{m}\right)^{2}}-f_{c} \leq 0
$$

where $\mathcal{P}$ is the equivalent stress, $r$ and $n$ are given in function of the tension and compression strengths $f_{c}$ and $f_{t}$ and the parts of the free energy density developed 
when the tension, $\left(\Psi_{t}^{0}\right)_{L}$, or compression, $\left(\Psi_{t}^{0}\right)_{c}$, limits are reached; these quantities are defined as

$$
\begin{aligned}
& \left(\Psi_{t, c}^{0}\right)_{L}=\sum_{i=1}^{3} \frac{\left\langle p_{p 0 i}^{m}\right\rangle \varepsilon_{n i}}{2 \rho_{0}}, \quad \Psi_{t}^{0}=\left(\Psi_{t}^{0}\right)_{L}+\left(\Psi_{c}^{0}\right)_{L} \\
& f_{t}=\left(2 \rho \Psi_{t}^{0} E_{0}\right)_{L}^{\frac{1}{2}}, \quad f_{c}=\left(2 \rho \Psi_{c}^{0} E_{0}\right)_{L}^{\frac{1}{2}}, \quad n=\frac{f_{c}}{f_{t}}, \quad r=\frac{\sum_{i=1}^{3}\left\langle P_{p 0 i}^{m}\right\rangle}{\sum_{i=1}^{3}\left|P_{p 0 i}^{m}\right|}
\end{aligned}
$$

A more general expression equivalent to that given in Equation (10a), Barbat et al. (1997), is given by

The function $\mathcal{G}(\mathcal{P})$ has the following general expression Oliver et al. (1990):

$$
\mathcal{G}(\chi)=1-\frac{\overline{\mathcal{G}}(\chi)}{\chi}=1-\frac{\chi^{*}}{\chi} e^{k\left(1-\frac{\chi^{*}}{\chi}\right)}
$$

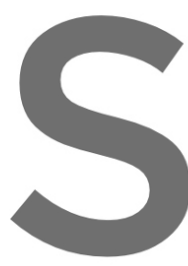

where the term $\chi=\chi^{*}$ and for is calibrated to energy of the material characteristic length of the fractured don

The evolution law for the internal damage variable
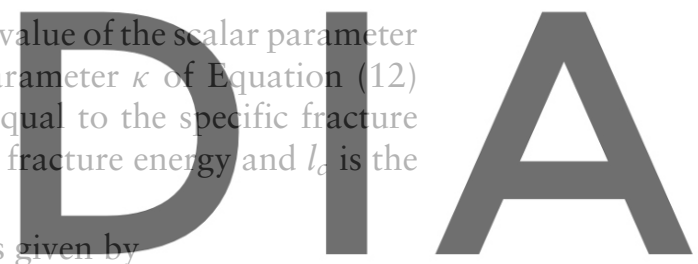

\section{Register for free at https: $\left(\begin{array}{l}\partial \bar{T} \\ \partial \mathcal{P}\end{array}\right.$}

where $\dot{\mu}=\dot{\mathcal{P}} \geq 0$ is the damage consistency parameter Mata et al. (2007b). Finally, the Kuhn-Tucker relations: (a) $\dot{\mu} \geq 0$, (b) $\overline{\mathcal{F}} \leq 0$, (c) $\dot{\mu} \overline{\mathcal{F}}=0$, have to be employed to derive the unloading-reloading conditions i.e. if $\overline{\mathcal{F}}<0$ the condition (c) imposes $\dot{\mu}=0$; on the contrary, if $\dot{\mu}>0$ then $\overline{\mathcal{F}}=0$.

\section{I.I Viscosity}

The rate dependent behavior is considered by using the Maxwell model. The FPK stress vector $\hat{P}_{1}^{m t}$ is obtained as the sum of a rate independent part $\hat{P}_{1}^{m}$, Equation (9), and a viscous component $\hat{P}_{1}^{m v}$ as

$$
\hat{P}_{1}^{m t}=\hat{P}_{1}^{m}+\hat{P}_{1}^{m v}=\mathcal{C}^{m v} \hat{\varepsilon}_{n}+\eta^{s m} \hat{\boldsymbol{S}}_{n}=(1-d) \mathcal{C}^{m e}\left(\hat{\varepsilon}_{n}+\frac{\eta}{E_{0}} \hat{\boldsymbol{S}}_{n}\right)
$$

where $\eta^{s m}=\eta / E_{0} \mathcal{C}^{m s}$ is the secant viscous constitutive tensor, $\mathcal{C}^{m v}=(1-d) \mathcal{C}^{m e}$, and the parameter $\eta$ is the viscosity. For the case of a completely damaged material $(d=1)$, 
the corresponding stresses are zero. The linearized increment of the FPK stress vector (material and co-rotated forms) are calculated as

$$
\Delta \hat{P}_{1}^{m t}=\mathcal{C}^{m v} \Delta \hat{\varepsilon}_{n}+\eta^{s m} \Delta \hat{S}_{n}, \quad \Delta\left[\hat{P}_{1}^{t}\right]=\mathcal{C}^{s v} \Delta\left[\hat{\varepsilon}_{n}^{\nabla}\right]+\eta^{s s} \Delta\left[\hat{s}_{n}\right]
$$

where $\mathcal{C}^{s v}=\boldsymbol{\Lambda} \mathcal{C}^{m v} \boldsymbol{\Lambda}^{T}$ and $\mathcal{C}^{s v}=\boldsymbol{\Lambda} \eta^{s s} \boldsymbol{\Lambda}^{T}$. The explicit form of the terms $\Delta \hat{S}_{n}$ and $\left[\hat{S}_{n}\right]$ can be consulted in reference Mata et al. (2008a). Finally, the material description of the tangent constitutive tensor $\mathcal{C}^{m v}$ considering the viscous effect is given by Barbat et al. (1997)

$$
\mathcal{C}^{m v}=\left(\mathbf{I}-\mathbf{D}^{m e}\right) \mathcal{C}^{m e}=\mathbf{I}-\left[\mathbf{I}+\frac{d \mathcal{G}}{d \mathcal{P}^{m}}\left(\hat{P}_{01}^{m}+\hat{P}_{1}^{m v 0}\right) \otimes \frac{\partial \mathcal{P}^{m}}{\partial \hat{P}_{01}^{m}}\right]
$$

\subsection{Plastic materials}

In the case of materials which can undergo non-reversible deformations the plasticity model formulated in the material configuration is used for predicting their mechanical response. Assuming small elastic, finite plastic deformations, an adequate form of the free energy density and analogous procedures as those for the damage model we have
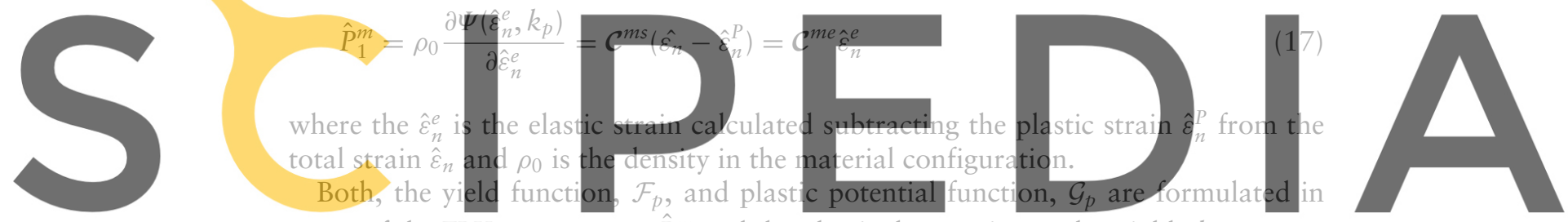

terms of the FPK stress vector $\hat{P}_{1}^{m}$ and the plastic damage internal variable $k_{p}$ as

where $\mathcal{F}_{p}\left(\hat{P}_{1}^{m}, k_{p}\right)$ is the equivalent stress, which is compared with the bardening function $f_{p}\left(\hat{P}_{1}^{m}, k_{p}\right)$ and $\mathcal{K}$ is a constant value, Oller et al. (1996a). In this work, $k_{p}$ constitutes a measure of the energy dissipated during the plastic process and, therefore, it is well suited for materials with softening and is defined by Oller et al. (1996b) as

$$
g_{f}^{P}=\frac{G_{f}^{P}}{l_{c}}=\int_{t=0}^{\infty} \hat{P}_{1}^{m} \cdot \dot{\varepsilon}_{n}^{P} d t, \quad 0 \leq\left[k_{p}=\frac{1}{g_{f}^{P}} \int_{t=0}^{t} \hat{P}_{1}^{m} \cdot \dot{\varepsilon}_{n}^{P} d t\right] \leq 1
$$

where $G_{f}^{P}$ is the specific plastic fracture energy of the material in tension and $l_{c}$ is the length of the fractured domain defined in analogous manner as for the damage model. The integral term in Equation (19) corresponds to the energy dissipated by means of plastic work.

The flow rules for the internal variables $\hat{\varepsilon}_{n}^{P}$ and $k_{p}$ are defined as

$$
\dot{\hat{\varepsilon}}_{n}^{P}=\dot{\lambda} \frac{\partial \mathcal{G}_{p}}{\partial \hat{P}_{1}^{m}}, \quad k_{p}=\dot{\lambda} \hat{\varrho}\left(\hat{P}_{1}^{m}, k_{p}, G_{f}^{P}\right) \cdot \frac{\partial \mathcal{G}_{p}}{\partial \hat{P}_{1}^{m}}=\hat{\varrho}\left(\hat{P}_{1}^{m}, k_{p}, G_{f}^{P}\right) \cdot \dot{\hat{\varepsilon}}_{n}^{P}
$$


where $\dot{\lambda}$ is the plastic consistency parameter and $\hat{\varrho}$ is the hardening vector, Oller et al. (1996a). Regarding the hardening function of Equation (18), the following evolution equation has been proposed:

$$
f_{p}\left(\hat{P}_{1}^{m}, k_{p}\right)=r \sigma_{t}\left(k_{p}\right)+(1-r) \sigma_{c}\left(k_{p}\right)
$$

where $r$ has been defined in Equation $(10 \mathrm{c})$ and the scalar functions $\sigma_{t}\left(k_{p}\right)$ and $\sigma_{c}\left(k_{p}\right)$ describe the evolution of the yielding threshold in uniaxial tension and compression tests, respectively.

As it is a standard practice in plasticity, the loading/unloading conditions are derived in the standard form from the Kuhn-Tucker relations formulated for problems with unilateral restrictions, i.e. (a) $\dot{\lambda} \geq 0$, (b) $\mathcal{F}_{p} \leq 0$ and (c) $\dot{\lambda} \mathcal{F}_{p}=0$. Starting from the plastic consistency condition $\dot{\mathcal{F}}_{p}=0$ and considering the flow rules it is possible to deduce the explicit form of $\dot{\lambda}$, as Oller et al. (1996a)
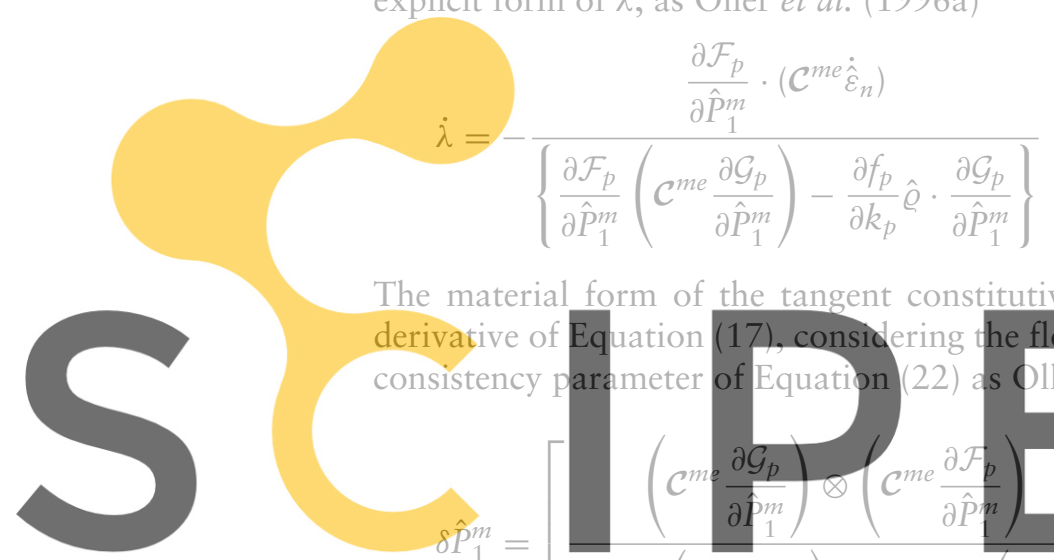

The material
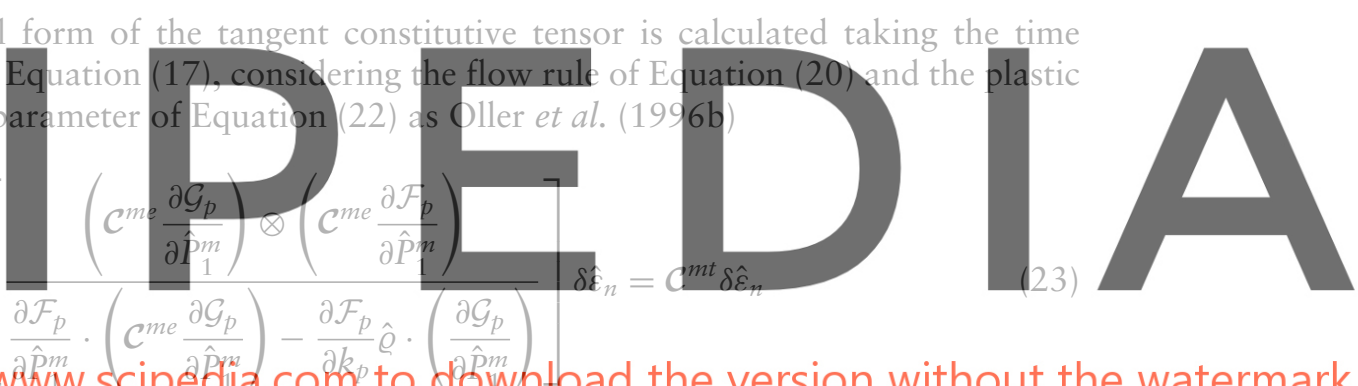

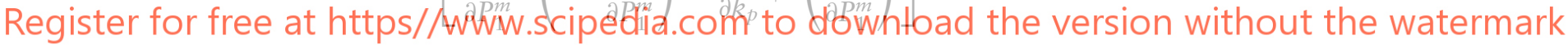

\subsection{Mixing theory for composites}

Each material point on the beam cross section is treated as a composite material according to the mixing theory, Oller et al. (1996a). The interaction between all the components defines the overall mechanical behavior of the composite at material point level. Supposing $N$ different components coexisting in a generic material point subjected to the same material strain $\hat{\varepsilon}_{n}$, we have the following closing equation: $\hat{\varepsilon}_{n} \equiv\left(\hat{\varepsilon}_{n}\right)_{1}=\cdots=\left(\hat{\varepsilon}_{n}\right)_{q}=\cdots=\left(\hat{\varepsilon}_{n}\right)_{N}$, which imposes the strain compatibility between components. The free energy density of the composite, $\bar{\Psi}$, is obtained as the weighted sum of the free energy densities of the $N$ components. The weighting factors correspond to the quotient between the volume of the $q^{\text {th }}$ component, $V_{q}$ and the total volume, $V$, such that $\Sigma_{q} k_{q}=1$.

The material form of the FPK stress vector $\hat{P}_{1}^{m}$ for the composite, including the participation of rate dependent effects, is obtained in analogous way as for simple materials i.e.

$$
\hat{P}_{1}^{m t}=\sum_{q} k_{q}\left(\hat{P}_{1}^{m}+\hat{P}_{1}^{m v}\right)_{q}=\sum_{q} k_{q}\left[(1-d) \mathcal{C}^{m e}\left(\hat{\varepsilon}_{n}+\frac{\eta}{E_{0}} \hat{S}_{n}\right)\right]_{q}
$$


where $\left(\hat{P}_{1}^{m}\right)_{q}$ and $\left(\hat{P}_{1}^{m v}\right)_{q}$ correspond the strain and rate dependent stresses of each one of the $N$ components. The material form of the secant and tangent constitutive tensors for the composite, $\overline{\mathcal{C}}^{m s}$ and $\overline{\mathcal{C}}^{m t}$, are obtained as, Oller et al. (1996a)

$$
\overline{\mathcal{C}}^{m s}=\sum_{q=1}^{N} k_{q}\left(\mathcal{C}^{m s}\right)_{q}, \quad \overline{\mathcal{C}}^{m t}=\sum_{q=1}^{N} k_{q}\left(\mathcal{C}^{m t}\right)_{q} \delta \hat{\varepsilon}_{n}
$$

where $\left(\mathcal{C}^{m s}\right)_{q}$ and $\left(\mathcal{C}^{m t}\right)_{q}$ are the material form of the secant and tangent constitutive tensors of the $q^{\text {th }}$ component.

\subsection{Constitutive relations for EDDs}

The constitutive law proposed for EDDs is based on a previous work of Mata et al. (2007a) which provides a versatile strain-stress relationship with the following general form:

$$
\bar{P}^{m}\left(\varepsilon_{1}, \dot{\varepsilon}_{1}, t\right)=\bar{P}_{1}^{m}\left(\varepsilon_{1}, t\right)=\bar{P}_{2}^{m}\left(\dot{\varepsilon}_{1}, t\right)
$$

where $\bar{P}^{m}$ is the average stress in the EDD, $\varepsilon_{1}$ the strain level, $t$ the time, $\dot{\varepsilon}_{1}$ the strain rate, $\bar{P}_{1}^{m}$ and $\bar{P}_{2}^{m}$ are the strain dependent and rate dependent parts of the stress, respectively. The model uncouples the total stress in viscous and non-viscous components, which correspond to a viscous dashpot device acting in parallel with a nonlinear hysteretic spring. The purely viscous component does not requires to be a linear function of the strain rate. Additionally, hardening, and variable elastic modulus can be reproduced. The response of the nonlinear hysteretic spring is obtained solving system of nonlinear differential equations depending on a set of parameters calibrated from experimental data. Details about the determination of the parameters and the integration algorithm can be reviewed in Mata et al. (2007a).

\section{Numerical implementation}

In order to obtain a Newton type numerical solution, the linearized form of the weak form of Equation (6) is required, which can be written as

$$
\mathcal{L}\left[G\left(\hat{\varphi}_{*}, \boldsymbol{\Lambda}_{*}, \hat{b}\right)\right]=G\left(\hat{\varphi}_{*}, \boldsymbol{\Lambda}_{*}, \hat{b}\right)=D G\left(\hat{\varphi}_{*}, \boldsymbol{\Lambda}_{*}, \hat{b}\right) \cdot \hat{p}
$$

where $\mathcal{L}\left[G\left(\hat{\varphi}_{*}, \boldsymbol{\Lambda}_{*}, \hat{b}\right)\right]$ is the linear part of the functional $G(\hat{\varphi}, \boldsymbol{\Lambda}, \hat{b})$ at the configuration defined by $(\hat{\varphi}, \boldsymbol{\Lambda})=\left(\hat{\varphi}_{*}, \boldsymbol{\Lambda}_{*}\right)$ and $\hat{p} \equiv(\Delta \hat{\varphi}, \Delta \hat{\theta})$ is an admissible variation. The term $G\left(\hat{\varphi}_{*}, \boldsymbol{\Lambda}_{*}, \hat{b}\right)$ supplies the unbalanced force and it is composed by the contributions of the inertial, external and internal terms; the differential $D G\left(\hat{\varphi}_{*}, \boldsymbol{\Lambda}_{*}, \hat{b}\right) \cdot \hat{p}$, gives the tangential stiffness, Simo \& Vu-Quoc (1986).

The linearization of the inertial and external components, $D G_{i n t} \cdot \hat{p}$ and $D G_{\text {ext }} \cdot \hat{p}$ gives the inertial and load dependent parts of the tangential stiffness, $K_{1 *}$ and $K_{P *}$, 
respectively, and they can be consulted in Simo \& Vu-Quoc (1986). The linearization of the internal term is calculated as, Mata et al. (2007b and 2008a)

$$
D G_{i n t}\left(\hat{\varphi}_{*}, \boldsymbol{\Lambda}_{*}, \hat{b}\right) \cdot \hat{p}=\int_{0}^{L}(\hat{b}^{T} \underbrace{\left[\begin{array}{cc}
0 & 0 \\
-\widetilde{n_{*}}\left[\frac{d}{d S}\right] & 0
\end{array}\right]}_{\left[n_{s *}\right]} \hat{p}+\hat{b}^{T}\left[\mathbf{B}_{*}\right]^{T}\left[\begin{array}{c}
\Delta \widehat{n}_{*} \\
\Delta \widehat{m}_{*}
\end{array}\right]) d S
$$

where the skew-symmetric tensor $\widetilde{\boldsymbol{n}}_{*}$ is obtained from $\hat{n}_{*},[d / d S] \hat{v}=[\mathrm{I}] \hat{v}, \forall \hat{v} \in \mathbb{R}^{3}$, the operator $\left[\boldsymbol{n}_{S_{*}}\right]$ contributes to the geometric part of the tangent stiffness and the operator $\left[\mathbf{B}_{*}\right]$ relates the admissible variation $b$ and the co-rotated variation of the strain vectors. The explicit expression for $\left[\mathbf{B}_{*}\right]$ can be found in Kapania (2003) and Simo \& Vu-Quoc (1986).

The estimation of the linearized form of the sectional force and moment vectors appearing in Equation (28) requires taking into account the linearized relation existing between $\hat{P}_{1}^{m}$, obtained using the mixing rule, and $\hat{\varepsilon_{n}}$. After integrating over the beam cross section, the following result is obtained for the linearized relation between cross sectional forces and the reduced strain measures Mata et al. (2007b and 2008a)

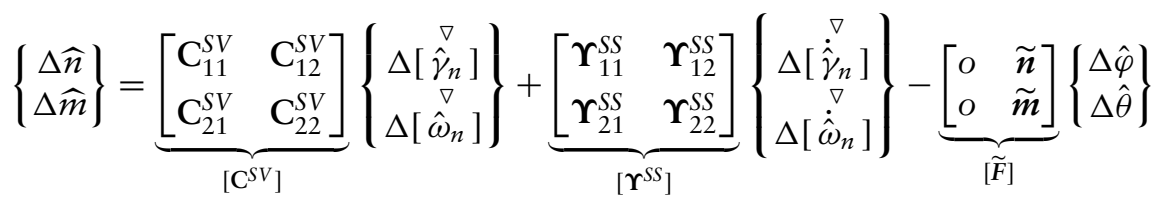

where $\tilde{m}$ is the skew-symmetric tensor obtained from $\widehat{m}, \mathrm{C}_{i j}^{S V}$ and $\Upsilon_{i j}^{S S},(i, j=1,2)$ are the spatial forms of the reduced tangential and viscous tangential constitutive tensors, which are rate dependent and can be consulted in Mata et al. (2008a). Finally, Equation (29) allows to rewrite Equation (28) as

$$
\begin{aligned}
D G_{i n t} \cdot \hat{p}= & \underbrace{\int_{0}^{L} \hat{b}^{T}\left[\mathbf{B}_{*}\right]^{T}\left[\mathbf{C}_{*}^{\mathrm{st}}\right]\left[\mathbf{B}_{*}\right] \hat{p} d S}_{K_{G *}}+\underbrace{\int_{0}^{L} \hat{b}^{T}\left[\mathbf{B}_{*}\right]^{T}\left[\boldsymbol{\Upsilon}_{*}^{\mathrm{st}}\right]\left[v_{*}\right] \hat{p} d S}_{K_{M *}} \\
& +\underbrace{\int_{0}^{L} \hat{b}^{T}\left(\left[\widetilde{\boldsymbol{n}}_{S *}\right]-\left[\mathbf{B}_{*}\right]^{T}\left[\widetilde{\mathbf{F}}_{*}\right]\right) \hat{p} d S}_{K_{V *}}
\end{aligned}
$$

where $K_{G *}, K_{M *}$ and $K_{v *}$, evaluated at the configuration $\left(\hat{\varphi}_{*}, \boldsymbol{\Lambda}_{*}\right)$, give the geometric, material and viscous parts of the tangent stiffness, which allows to rewrite Equation (27) as

$$
\mathcal{L}\left[G_{*}\right]=G_{*}+K_{1 *}+K_{M *}+K_{V *}+K_{G *}+K_{P *}
$$

The solution of the discrete form of Equation (30) by using the FE method follows identical procedures as those described by Simo \& Vu-Quoc (1986) for an iterative 
Newton-Rapson integration scheme and it will not be included here. Newmark's implicit time stepping algorithm has been chosen as integration method following the development originally proposed by Simo \& Vu-Quoc (1986). For the rotation part the time-stepping procedure takes place in $S O(3)$ and the basic steps, as well as the iterative update algorithm for the strain and strain rate vectors are given in Mata et al. (2007b and 2008a).

\section{I Cross sectional analysis}

The cross section analysis is carried out expanding each integration point on the beam axis in a set of integration points located on each fiber on the cross section. The cross section is meshed into a grid of quadrilaterals, each of them corresponding to a fiber oriented along the beam axis. The geometry of each quadrilateral is described by means of normalized bi-dimensional shape functions and several integration points can be specified according to a selected integration rule. The average value of a quantity, [•], for example, the components of FPK stress vector or the tangential tensor existing on a quadrilateral, are

$$
[\bullet]=\frac{1}{A_{c}} \int_{A_{c}}[\bullet] d A_{c}=\frac{1}{A_{c}} \sum_{p=1}^{N_{p}} \sum_{q=1}^{N_{q}}[\bullet]\left(y_{p}, z_{p}\right) J_{p q} W_{p} q
$$

where $A c$ is the area of the quadrilateral, $N_{p}$ and $N_{q}$ are the number of integration points in the two directions of the normalized geometry, $[\bullet]\left(y_{p}, z_{q}\right)$ is the value of the quantity [•] existing on a integration point with coordinates $\left(y_{p}, z_{q}\right)$ with respect to the reference beam axis, $J_{p q}$ is the Jacobian of the transformation between normalized coordinates and cross sectional coordinates and $W_{p q}$ are the weighting factors. Two additional integration loops are required. The first one runs over the quadrilaterals and the second loop runs over each simple material associated to the composite of the quadrilateral. More details can be consulted in Mata et al. (2007).

\section{Damage indices}

A measure of the damage level of a material point can be obtained as the ratio of the existing stress level to its elastic counterpart. Following this idea, it is possible to define the fictitious damage variable $\breve{D}$ as, Barbat et al. (1997)

$$
\sum_{i=1}^{3}\left|P_{1 i}^{m}\right|=(1-\check{D}) \sum_{i=1}^{3}\left|P_{1 i 0}^{m}\right| \rightarrow \breve{D}=1-\frac{\sum_{i=1}^{3}\left|P_{1 i}^{m}\right|}{\sum_{i=1}^{3}\left|P_{1 i 0}^{m}\right|}
$$

where $\left|P_{1 i}^{m}\right|$ and $\left|P_{1 i 0}^{m}\right|$ are the absolute values of the components of the existing and elastic stress vectors, respectively. Initially, the material remains elastic and $D=0$, but when all the energy of the material has been dissipated $\left|P_{1 i}^{m}\right| \rightarrow 0$ and $\breve{D} \rightarrow 1$. Equation (33) can be extended to consider elements or even the whole structure by means of integrating over a finite volume as follows:

$$
\check{D}=1-\frac{\int_{V_{p}}\left(\Sigma_{i}\left|P_{1 i}^{m}\right|\right) d V_{p}}{\int_{V_{p}}\left(\Sigma_{i}\left|P_{1 i 0}^{m}\right|\right) d V_{p}}
$$


where $V_{p}$ is the volume of the part of the structure. Equation (34) is easily implemented in a standard FEM code without requiring large extra memory storage.

\section{Numerical examples}

\section{I Experimental-numerical comparative study of a scaled RC building model}

The first example corresponds to the comparison between the numerical simulation obtained by means of the proposed formulation and the experimental data obtained by $\mathrm{Lu}(2002)$ for the seismic analysis of a scaled model (1:5.5) of a benchmark regular bare frame (BFR). The structure was designed for a ductility class medium in accordance with the Eurocode 8 (2001) with a peak ground acceleration of $0,3 \mathrm{~g}$ and a soil profile A. Details about loads, geometry, material properties and distribution of steel reinforcements can be consulted in the same publication. In the experimental program, the structure was subjected to several scaled versions of the N-S component of the El Centro 1940 earthquake record. Four quadratic elements with two Gauss integration points were used for each beam and column. Cross sections were meshed into a grid of 20 equally spaced layers. Longitudinal steel reinforcements were included in the external layers as part of a composite material. The fracture energy of the damage model used for concrete was modified to take into account the confining effect of transversal stirrups, Mata et al. (2007b). A tension to compression ratio of 10 was used for concrete and 1 for steel. In the numerical simulations, the model is subjected to a push-over analysis. Static forces derived from the inertial contribution of the masses are applied at the floor levels considering an inverted triangular distribution. A relationship between the measured base shear and the top lateral displacement is given by Lu (2002) for each seismic record. This curve is compared in Figure 3 with

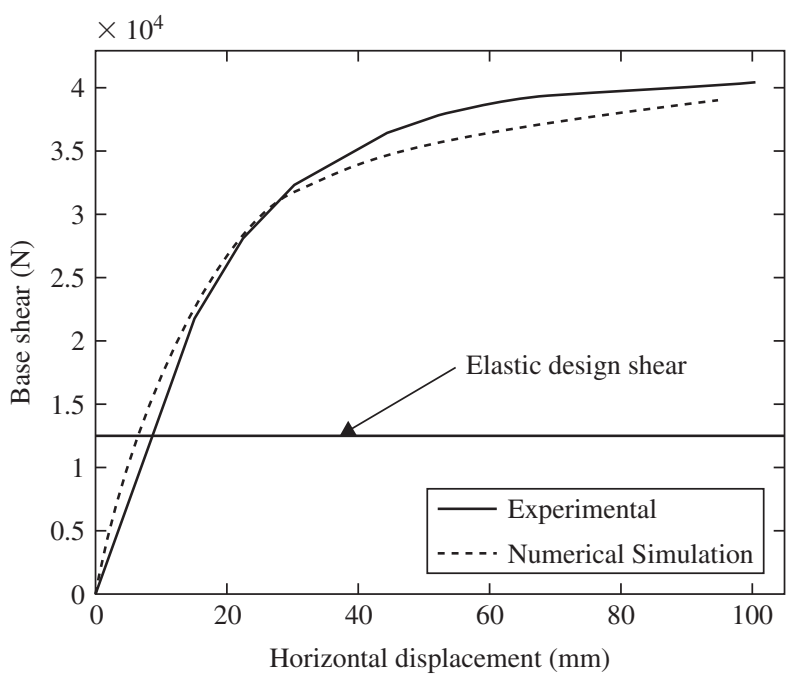

Figure 3 Capacity curves: Comparison between results from numerical pushover and experimental tests. 
the capacity curve obtained by using the numerical push-over analysis. It is possible to see that the push-over analysis gives a good approximation for the global maximum response and, therefore, it constitutes a suitable numerical procedure for estimating the expected nonlinear properties of structures subjected lo seismic actions. In the same figure, it is possible to appreciate that in both, the numerical simulation and the experimental cases, the characteristic values of the structure; that is, global ductility level, elastic limit and overstrength, are similar. Figure 4 shows a comparison between the distribution of cross sectional damage predicted numerically and the map of fissures obtained after the application of several shaking table tests.

In this case, the proposed damage index along with the geometric and constitutive formulation used for beams is able to reproduce the general failure mechanism of the structure where dissipation is mainly concentrated in the beam elements.

\subsection{Seismic response of a precast RC building with EDDs}

The nonlinear seismic response of a typical precast RC industrial building shown in Figure 5 is studied. The building has a bay width of $24 \mathrm{~m}$ and $12 \mathrm{~m}$ of inter-axes length. The story height is $10 \mathrm{~m}$. The concrete of the structure is $\mathrm{H}-35,(35 \mathrm{MPa}$, ultimate compression), with an elastic modulus of $29.000 \mathrm{MPa}$. It has been assumed a Poisson coefficient of 0.2. The ultimate tensile stress for the steel is $510 \mathrm{MPa}$. This figure also shows some details of the steel reinforcement of the cross sections. The dimensions of the columns are $60 \times 60 \mathrm{~cm}^{2}$. The beam has an initial high of $60 \mathrm{~cm}$ on the supports and $160 \mathrm{~cm}$ in the middle of the span. The permanent loads considered are $1050 \mathrm{~N} / \mathrm{m}^{2}$ and the weight of upper half of the closing walls with $432,000 \mathrm{~N}$. The input acceleration is the same as in example 6.1.

The half part of the building is meshed using 4 quadratic elements with two Gauss integration points for the resulting beam and column. The cross sectional grid of fibers is shown in Figure 6. One integration point is used for each quadrilateral.

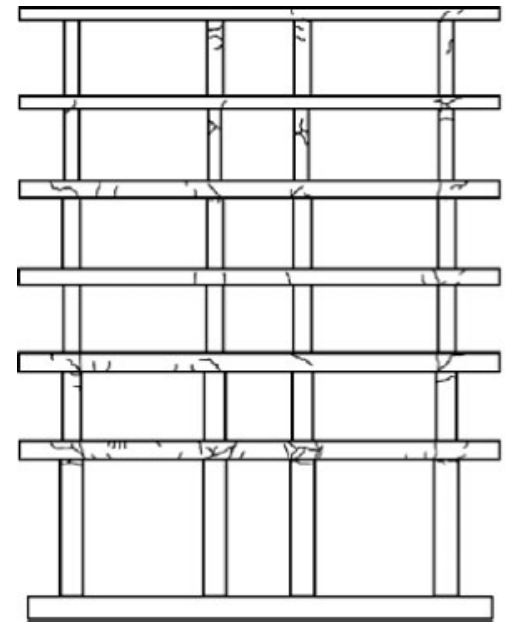

(a)

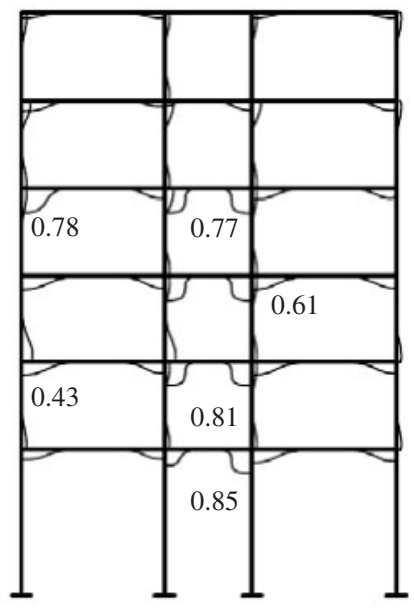

(b)

Figure 4 Damage. (a) Experimental: Map of fissures. (b) Numerical: Cross sectional damage index. 


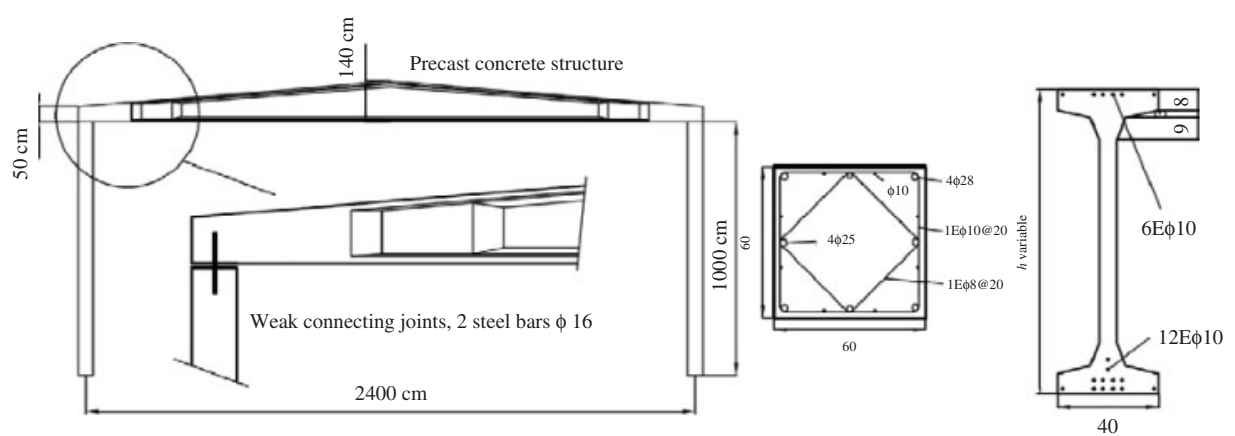

Figure 5 Description of the structure.
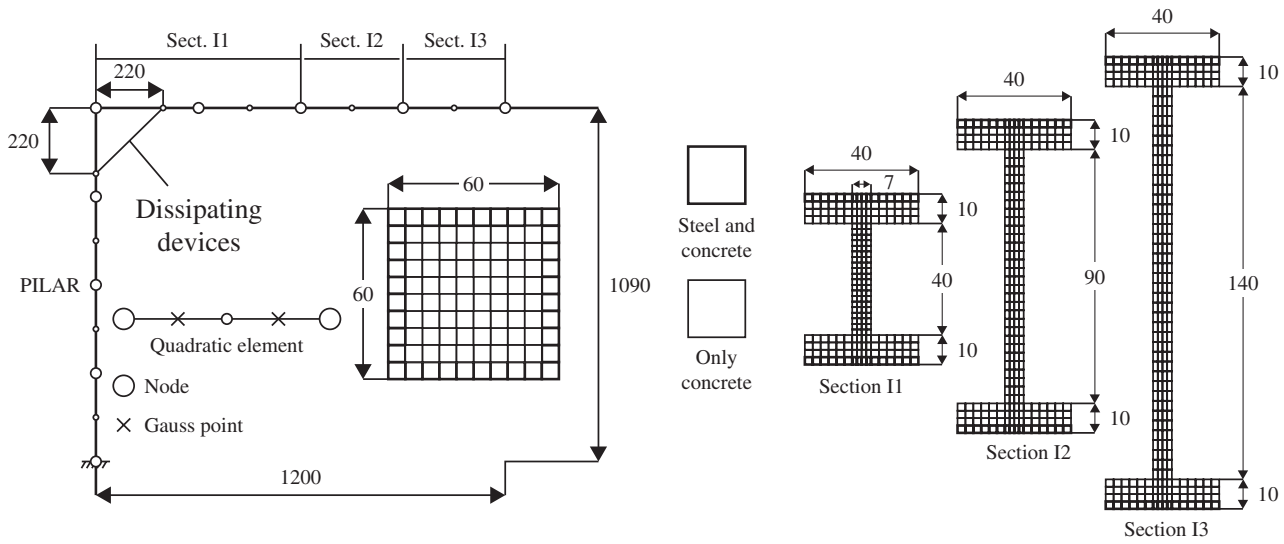

Figure 6 Half part of 2D precast industrial frame. I: Normal frame 2: Energy dissipating devices incorporated (diagonal elements). 3: Numerical model of column and beam cross sections.

The EED was simulated by means of employing the previously described model reproducing a plastic dissipative mechanism. The properties of the device were designed for yielding with an axial force of $150.000 \mathrm{~N}$ and for a relative displacement between the two ending nodes of $1.5 \mathrm{~mm}$. The length of the devices was of $2.0 \mathrm{~m}$. The results of the numerical simulations allow seeing that the employment of plastic EDDs contributes to improve the seismic behavior of the structure for the case of the employed acceleration record. Figure $7 \mathrm{a}$ shows the hysteretic cycles obtained from the lateral displacement of the upper beam-column joint and the horizontal reaction (base shear) in the columns for the structure with and without devices. It is possible to appreciate that the non-controlled structure (bare frame) presents greater lateral displacements and more structural damage, (greater hysteretic area than for the controlled case). Figure $7 \mathrm{~b}$ shows the hysteretic cycles obtained in the EDD, evidencing that part of the dissipated energy is concentrated in the controlling devices, as expected. 


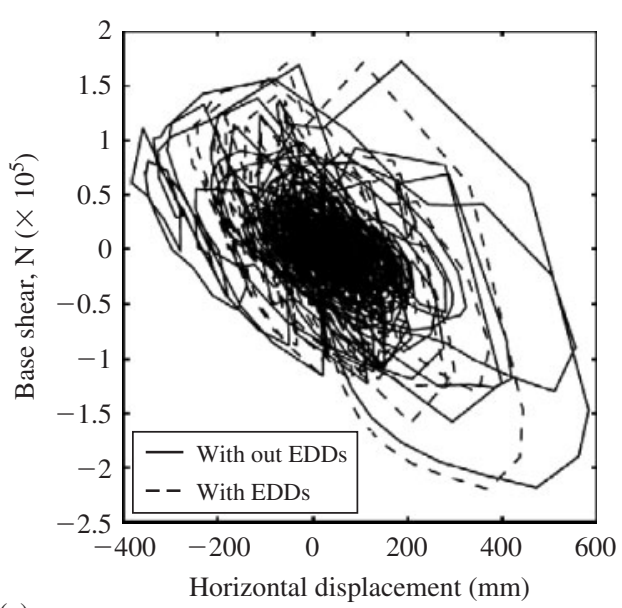

(a)

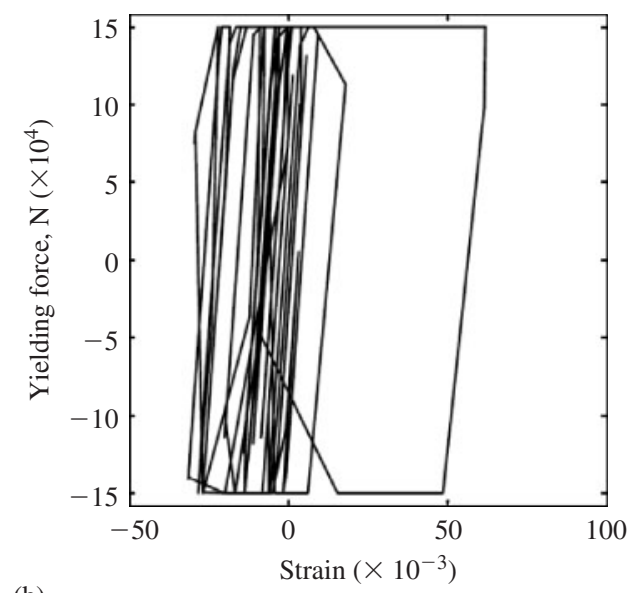

(b)

Figure 7 (a) base shear-displacement relationship. (b) Hysteretic cycles in the EDD.

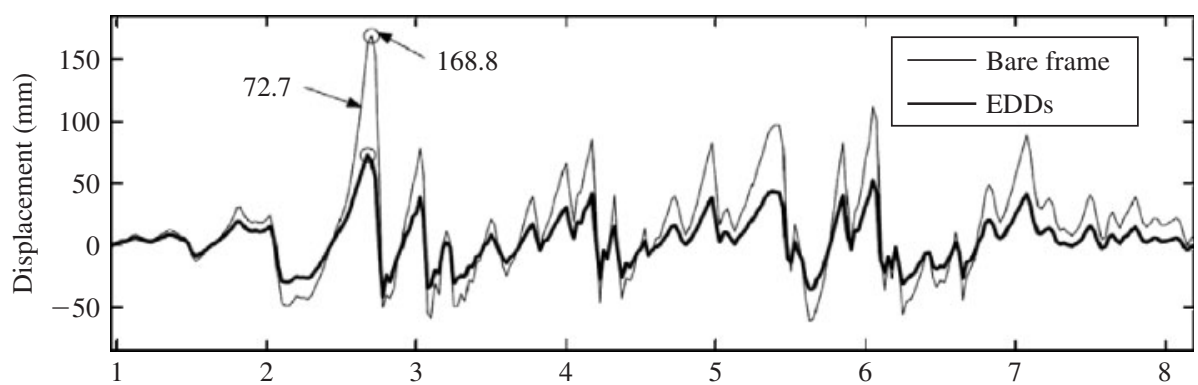

Figure 8 Time history of the top horizontal displacement.

Figure 8 shows the time history response of the horizontal displacement of the upper beam-column joint. A reduction of approximately $40 \%$ is obtained for the maximum lateral displacement when compared with the bare frame. Acceleration and velocity are controlled in the same way, but only 10 and $5 \%$ of reduction is obtained. A possible explanation for the limited effectiveness of the EDD is that the devices only contribute to increase the ductility of the beam-column joint without alleviating the base shear demand on the columns due to the dimensions of the device and its location in the structure. By other hand, joints are critical points in precast structures and therefore, the employment of EDDs combined with a careful design of the columns can help to improve their seismic behavior.

\section{Conclusions}

In this work, a geometrically exact formulation for initially curved beams has been extended to consider arbitrary distributions of composite materials on the cross 
sections in the seismic case. The consistent linearization of the weak form of the momentum balance equations considers the constitutive nonlinearity with rate dependent effects. The resulting model is implemented in a displacement based FEM code. An iterative Newton-Rapson scheme is used for the solution of the discrete version of the linearized problem. A specific element for EDD is developed, based on the beam model but releasing the rotational degrees of freedom.

Each material point of the cross section is assumed to be composed of several simple materials with their own constitutive laws. The mixing rule is used to describe the resulting composite. Viscosity is included at constitutive level by means of a Maxwell model. Beam cross sections are meshed into a grid of quadrilaterals corresponding to fibers directed along the beam axis. Two additional integration loops are required at cross sectional level in each integration point to obtain the reduced quantities. Local and global damage indices have been developed based on the ratio between the viscoelastic and the nonlinear stresses.

The present formulation is validated by means of two numerical examples, which include the comparison with existing experimental data and the study of the seismic response of a precast reinforced concrete structure with EDDs.

\section{Acknowledgement}

This research was partially supported by the European Commission, CEE-FP6 Project FP650544(GOCE) "Risk Mitigation for Earthquakes and Landslides (LESSLOSS)", by the Spanish Government (Ministerio de Educación y Ciencia), project BIA2003-08700C03-02 "Numerical simulation of the seismic behaviour of structures with energy dissipation devices", project MAT2003-09768-C03-02 "Delamination of reinforced matrix composites (DECOMAR)" project BIA2005-06952 "Study of composite materials for design, reinforcement and retrofit of civil engineering structures (RECOMP)" by the Spanish Government (Ministerio de Fomento) "Numerical simulation methodology for the reinforced concrete behavior structures reinforced with composite materials", and by the collaboration framework between CIMNE and AIRBUS, project PBSO-13-06 "Innovative finite element methods for non linear analysis of composite structures (FEMCOM)". All this support is gratefully acknowledged.

\section{References}

Aiken, I. 1996. Passive energy dissipation hardware and applications. Proceedings, Lo Angeles County and Seaosc Symposium on Passive Energy Dissipation Systems for New and Existing Buildings, Los Angeles.

Antman, S.S. 1991. Nonlinear Problems of Elasticity, Springer-Verlag, 1991.

Barbat, A.H. Oller, S. Hanganu, A. \& Oñate, E. 1997. Viscous damage model for Timoshenko beam structures. International Journal for Solids and Structures; 34(30):3953-3976.

Bayrak, O. \& Sheikh, S. A. 2001. Plastic hinge analysis. Journal of Structural Engineering; 127(9):1092-1100.

Clark, P. Aiken, I. Ko, E. Kasai K. \& Kimura, I. 1999. Design procedures for buildings incorporating hysteretic seismic devices. Proceedings, 68th Annual Convention Santa Barbara, California Structural Engineering Association of California. 
Connor, J.J. Wada, A. Iwata, M. \& Huang Y.H. 1997. Damage-controlled structures. I: Preliminary design methodology for seismically active regions. Journal of Structural Engineering; 123(4):423-431.

EC8, Eurocode No 8. 2001. Design of structures for earthquake resistance. European Committee for standardization, 3rd Draft, prEN 1998-1-1.

Federal Emergency Management Agency. 2000. Prestandard and Commentary for the Seismic Rehabilitation of Buildings, Report 356, Washington, DC: FEMA.

Federal Emergency Management Agency. NEHRP Recommended Provisions for Seismic Regulations for New Buildings and Other Structures. 2000. Report 368, Washington, DC: FEMA.

Fu, Y. \& Kasai, K. 1998. Comparative study of frames using viscoelastic and viscous dampers. Journal fo Structural Engineering; 124(5):513-522.

Hanganu, A. Oñate E. \& Barbat, A.H. 2002. Finite element methodology for local/global damage evaluation in civil engineering structures. Conputers and Structures; 80:1667-1687.

Hanson, R.D. Aiken, I.D. Nims, D.K. Ritchter, P.J. \& Batchman, R.E. 1993. State of the art and state of the practice in seismic engineering dissipation. Proceedings, ATC-17-1. Seminar on seismic isolation, passive energy dissipation and active control. Applied Technology Council, San Francisco, California.

I. Computer Methods in Applied Mechanic and Engineering; 49:55-70.

Ibrahimbegovic, A. 1995. On finite element implementation of geometrically nonlinear Reissners beam theory: three-dimensional curved beam elements. Computer Methods in Applied Mechanics and Engineering; 122:11-26.

Kapania, R.K. \& Li, J. 2003. On a geometrically exact curved/twisted beam theory under rigid cross-section assumption. Computational Mechanics; 30:428-443.

Kasai, K. Fu, Y. \& Watanabe, A. 1998. Passive control systems for seismic damage mitigation. Journal of Structural Engineering; 124:(5):501-512.

Kumar, P. Nukala, V.V. \& White, D.W. 2004. A mixed finite element for threedimensional nonlinear analysis of steel frames. Computer Methods in Applied Mechanic and Engineering; 193(5):2507-2545.

Lin, W.H. \& Chopra, A.K. 2003. Asymmetric one-storey elastic system with non-linear viscous and viscoelastic dampers: Earthquake response. Earthquake Engineering and Structural Dynamics; 32:555-577.

Lin, Y.Y. \& Chang, K.C. 2003. Study on damping reduction factors for buildings under earthquake ground motions. Journal of Structural Engineering, JEE; 129(2):206-214.

Lu, Y. 2002. Comparative study of seismic behavior of multistory reinforced concrete framed structures. Journal of Structural Engineering, JEE; 128(2):169-178.

Mata, P. Oller, S. Barbat, A.H. \& Boroschek, R. 2006. Numerical code for seismic analysis of structures incorporating energy dissipating devices. First European Conference on Earthquake Engineering and Seismology, ECEES, Geneva, Switzerland.

Mata, P. Boroschek, R. Oller, S. \& Barbat, A.H. 2007a. High damping rubber model for energy dissipating devices, Journal of Earthquake Engineering; 11(2):231-256.

Mata, P. Oller, S. \& Barbat, A.H. 2007b. Static analysis of beam structures under nonlinear geometric and constitutive behavior, Computer Methods in Applied Mechanics and Engineering; 196:4458-4478.

Mata, P. Oller, S. \& Barbat, A.H. 2008a. Dynamic analysis of beam structures considering geometric and constitutive nonlinearity, Computer Methods in Applied Mechanics and Engineering; 197:857-878.

Mata, P. Oller, S. Barbat, A.H. \& Boroschek, R. 2008b. Computational models for the seismic response of reinforced concrete buildings with energy dissipating devices, Archives of Computational Methods in Engineering, (Accepted).

Mata, P. Oller, S. Barbat, A.H. \& Boroschek, R. 2008c. Nonlinear seismic analysis of RC structures with energy dissipating devices, International Journal for Numerical Methods in Engineering, (Submitted). 
Oliver, J. Cervera, M. Oller, S. \& Lubliner, J. 1990. Isotropic damage models and smeared crack analysis of concrete. Proceedings 2nd ICCAADCS, Zell Am See, Austria, Pineridge Press; 2:945-958.

Oller, S. Oñate, E. \& Miquel, J. 1996a. Mixing anisotropic formulation for the analysis of composites, Communications in Numerical Methods in Engineering. 12 471-482.

Oller, S. Oñate, E. Miquel, J. \& Botello, S. 1996b. A plastic damage constitutive model for composites materials. International Journal of Solids and Structures; 33(17):2501-2518.

Reissner, E. 1973. On one-dimensional large-displacement finite-strain beam theory. Studies inApplied Mathematics; LII, 287-295.

Shen, K.L. \& Soong, T.T. 2005. Design of energy dissipation devices based on concept of damage control. Journal of Structural Engineering; 122(1):76-82.

Simo, J.C. \& Vu-Quoc, L. 1986. A three-dimensional finite-strain rod model. Part II: Computational aspects. Computer Methods in Applied Mechanics and Engineering; 58:79-116.

Simo, J.C. \& Vu-Quoc, L. 1988. On the dynamics in space of rods undergoing large motions A geometrically exact approach. Computer Methods in Applied Mechanics and Engineering; 66:125-161.

Simo, J.C. 1985. A finite strain beam formulation. The three-dimensional dynamic problem. Part

Simo, J.C. Hjelmstad, K.D. \& Taylor, R.L. 1984. Numerical formulations of elastoviscoplastic response of beams accounting for the effect of shear. Computer Methods in Applied Mechanics and Engineering; 42:301-330.

Soong, T.T. \& Dargush, G.F. 1997. Passive Energy Dissipation Systems in Structural Engineering; John Wiley \& Sons Ltda. 